\title{
¿

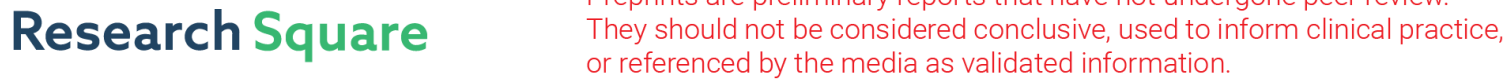 \\ Clinical Relevance of Covid-19 in the Second Half of Pregnancy
}

\section{Marta RUGGIERO}

Università degli Studi di Milano: Universita degli Studi di Milano

\section{Edgardo Somigliana ( $\nabla$ dadosomigliana@yahoo.it )}

Università degli Studi di Milano: Universita degli Studi di Milano https://orcid.org/0000-0002-02230032

\section{Beatrice TASSIS}

La Fondazione IRCCS Ca\' Granda: Fondazione IRCCS Ca' Granda Ospedale Maggiore Policlinico

\section{Letizia LI PIANI}

Università degli Studi di Milano: Universita degli Studi di Milano

\section{Sara Uceda Renteria}

Fondazione irccs ca' granda ospedale maggiore policlinico

\section{Giussy BARBARA}

La Fondazione IRCCS Ospedale Maggiore Policlinico: Fondazione IRCCS Ca' Granda Ospedale Maggiore Policlinico

\section{Giovanna LUNGHI}

FOndazione irccs ca' granda ospedale maggiore policlinico

\section{Enrico FERRAZZI}

Università degli studi di milano

\section{Research article}

Keywords: Covid-19, Sars-Cov-2, pregnancy, antibodies

Posted Date: February 25th, 2021

DOI: https://doi.org/10.21203/rs.3.rs-271806/v1

License: (9) This work is licensed under a Creative Commons Attribution 4.0 International License. Read Full License

Version of Record: A version of this preprint was published at BMC Pregnancy and Childbirth on July 12th, 2021. See the published version at https://doi.org/10.1186/s12884-021-03985-1. 


\section{Abstract}

Background: Evidence on the outcome of Covid-19 in pregnancy is generally reassuring but yet not definitive.

Methods: To specifically assess the impact of Covid-19 in the second half of pregnancy, we prospectively recruited 315 consecutive women delivering in a referral hospital located in Lombardy, Italy in the early phase of the epidemic. Restriction of the recruitment to this peculiar historical time period allowed to exclude infections occurring early in pregnancy and to limit the recall bias. All recruited subjects underwent a nasopharyngeal swab to assess the presence of Sars-Cov-2 using Real-time PCR. In addition, two different types of antibodies for the virus were evaluated in peripheral blood, those against the spike proteins S1 and S2 of the envelope and those against the nucleoprotein of the nucleocapsid. Women were considered to have had Covid-19 in pregnancy if at least one of the three assessments was positive.

Results: Overall, 28 women had a diagnosis of Covid-19 in pregnancy (8.9\%). Women diagnosed with the infection were more likely to report one or more episodes of symptoms suggestive for Covid-19 $(n=11$, $39.3 \%)$ compared to unaffected women $(n=39,13.6 \%)$. The corresponding OR was 4.11 (95\%Cl: $1.79-$ 9.44). Symptoms significantly associated with Covid-19 in pregnancy included fever, cough, dyspnea and anosmia. Only one woman necessitated intensive care. Pregnancy outcome in women with and without Covid-19 did not also differ.

Conclusions: Covid-19 is asymptomatic in three out of five women in the second half of pregnancy and is rarely severe. In addition, pregnancy outcome may not be significantly affected.

\section{Background}

The Severe Acute Respiratory Syndrome Coronavirus type-2 (SARS-Cov-2) was first identified in the area of Wuhan, China, at the end of 2019 but then rapidly spread globally in the beginning of 2020 . In Lombardy, a region of Northern Italy that was particularly hit by the epidemic in the early phase, the first case was identified on February $20^{\text {th }}, 2020$ [1]. SARS-Cov-2 causes a potentially fatal infection named Covid-19 (Coronavirus Disease - 2019). Most common symptoms include fever, cough, sore throat, malaise, myalgia, gastrointestinal symptoms, anosmia and ageusia [2]. In more advanced conditions the subject can experience severe dyspnea and respiratory failure requiring admission in intensive care units [3]. The fatality rate varies widely in the literature (1-22\%) and depends on the clinical phase of the disease, gender, age and predisposing conditions such as diabetes, hypertensive disorders and obesity $[3,4]$.

Albeit still limited, there is evidence that SARS-Cov-2 can cause life-threatening situations also in pregnancy, even if fatal cases seem rare [5-9]. However, this evidence was mainly drawn from case series or selected referral centers, a study design that could overestimate the detrimental effects of SARS-Cov- 2 . Indeed, asymptomatic or poorly symptomatic women are more likely to go undetected, thus boosting the 
epidemiological relevance of Covid-19 related complications. To assess the clinical relevance of these complications in pregnancy one needs to know the real denominator, i.e. the total number of women being infected with the virus.

The use of antibodies could provide more informative evidence on this issue because their presence could reveal a past infection also in asymptomatic cases. Two different types of antibodies can currently be tested, those against the glycoproteins of the virus envelope and those against the nucleoproteins. The former are generally considered neutralizing while the role of the latter in controlling virus replication is not yet established. Both were shown to be valuable markers of infection [10].

To shed more light on Covid-19 in pregnancy, we prospectively recruited consecutive women delivering in a large hospital located in Milan, Lombardy, in the early phase of the epidemic. All women underwent a nasopharyngeal swab to investigate the presence of the virus and provided a blood sample to assess the presence of antibodies against SARS-Cov-2. The primary aim was to determine the rate of pregnant women who were exposed to Covid-19 during pregnancy. Secondary aims included the clinical presentation of the infection and the possible impact on pregnancy outcome. To note, the recruitment period lasted from April $7^{\text {th }}$ to May $6^{\text {th }}$ and thus offered us the unique opportunity to study women who could be exposed to SARS-Cov-2 exclusively during the second part of pregnancy.

\section{Methods}

The study took place at the Fondazione IRCCS Ca' Granda Ospedale Maggiore Policlinico, a hospital located in Milan, in the Italian region of Lombardy. The hospital ensures about 5,000 deliveries per year and was committed by the local health and governmental officials to be a referral hospital also for Covid19 in pregnancy. All women delivering between April $7^{\text {th }}$ to May $06^{\text {th }}, 2020$ were consecutively considered for study entry. Exclusion criteria were as follows: 1) pregnancy termination before 20 weeks' gestation (miscarriages), 2) referral from other hospitals because of Covid-19 diagnosis. This criterion was introduced to prevent confounders due to referrals. Eligible women were invited to participate the day after delivery. Women agreeing to be recruited signed an informed consent. They were requested to fill a specific questionnaire and provided a blood sample that was centrifuged at 3,500 rpm for 8 minutes at room temperature and stored at $-20^{\circ} \mathrm{C}$ until assayed for the presence of antibodies. For the nasopharyngeal swab for Sars-Cov-2, we relied on the ascertainment that was systematically performed at hospital admission. The study was approved by the local Institutional Review Board (Comitato Etico Milano Area 2, N. 356_2020) and we had the permission to collect the data from the hospital. All research was performed in accordance with the guidelines of the International Conference of Harmonization of Good Clinical Practice (ICH/GCP) and related regulations, including the Helsinki declaration of June 1964 and its adaptation done by the World Medical Association General Assembly in Seoul in 2008.

Collected information included general medical and obstetrics history as well as the pregnancy outcome of the index pregnancy. Patients' charts were used to obtain this information. The questionnaire that women had to fill evaluated the presence of symptoms suggestive for Covid-19 over the last three 
months. More than one episode of symptoms could be reported. The specific items included in the questionnaire are illustrated in details elsewhere [11]. Briefly, they include the presence of fever ( $\geq 37.5$ ${ }^{\circ} \mathrm{C}$ ), cough, sore throat, rhinitis, headache, diarrhea, vomit, dyspnea (including tachypnea), asthenia, myalgia, anosmia and ageusia. In addition, women were asked if they were exposed to higher risk of infection for job situations (health workers or laboratory technicians) or contacts with persons with a definitive diagnosis of Covid-19 or cohabitant with persons doing a job at risk or having symptoms suggestive for Covid-19.

Evaluation of Covid-19 in the cohort was based on three determinations: identification of the specific RNA on a nasopharyngeal swab and research of Immunoglobuline-type $\mathrm{G}(\mathrm{IgG})$ using two different tests. Women were considered to have had Covid-19 in pregnancy if at least one of the assessments was positive The presence of the virus in the nasopharyngeal swab was investigated using the multiplex realtime (RT)-PCR for the qualitative detection of three target genes of SARS-CoV-2 (Allplex 2019-nCov Assay, Seegene, Seoul, Korea): SARS-CoV-2 nucleocapsid proteins and RNA polymerase gene fragments (both specific for Sars-Cov-2), and envelope gene fragment for detection of the sarbecovirus subgenus family that includes SARS-CoV-2. The assay has a full-process negative control, positive control and internal control. The first serologic test was a chemiluminescence (CLIA) immunoassay (LIAISON®, Diasorin, Saluggia - Italy) used for the quantitative detection of IgG antobodies against S1 and S2 antigens of SARS-CoV-2. The S1 and S2 proteins are derived from SARS-CoV-2 spike protein that is responsible for entry into the host cell. Antibodies against these two targets are neutralizing. The test has a sensitivity and specificity of $97.4 \%$ and $98.5 \%$, respectively. The second test was an electrochemiluminescence (ECLIA) immunoassay (Elecsys ${ }^{\circledR}$, Roche Diagnostics, Mannheim, Germany) that uses a recombinant nucleoprotein representing the nucleocapsid antigen for the qualitative determination of antibodies against SARS-CoV-2. This nucleoprotein antigen is internal to the envelope. The test has a specificity greater than $99.8 \%$ and a sensitivity of $100 \%$.

Data analyses were performed using the Statistical Package for Social Sciences (SPSS 23.0, IL, USA). Women were considered to be affected if at least one of the three assessments resulted positive for SarsCov-2. The sample size (at least 250 subjects) was calculated expecting up to $20 \%$ of women being affected and aiming at a precision in the estimated prevalence of $+/-5 \%$. A binomial distribution model was used to determine the $95 \%$ Confidence Interval $(\mathrm{Cl})$ of the most relevant proportions. Data was compared using Student $t$ Test, Fisher exact test or Chi Square test as appropriate. P values below 0.05 were considered statistically significant.

\section{Results}

Three hundred eighty-five women delivered during the study period. Twelve were excluded because they were referred from other hospitals for Covid-19. Thirty-five were not recruited because of violation of the study protocol (the study was not proposed). Twenty-three women refused to participate. Three hundred fifteen women were ultimately enrolled. Overall, 28 women had a diagnosis of Covid-19 (8.9\%, 95\% Cl: 6.212.5\%). Specific rates according to the test used are reported in Table 1. Details on the concordance 
among tests are illustrated in Table 2. To note, among the 24 women who were detected with antibodies against Sars-Cov-2, 17 (71\%) were positive to both tests used, three (12\%) were positive only for antibodies against the envelop and four (17\%) only to those against the nucleoprotein. Baseline characteristics of women who did and did not have Covid-19 are shown in Table 3. The two groups did not significantly differ for any of these characteristics.

Risk factors for Covid-19 are shown in Table 4. Symptoms significantly associated with Covid-19 included fever, cough, dyspnea and anosmia. A trend emerged also for ageusia (Table 4). Women diagnosed with the infection were more likely to report one or more episodes of symptoms suggestive for Covid-19 ( $n=11,39.3 \%)$ compared to unaffected women $(n=39,13.6 \%)(p=0.001)$. The corresponding Odds Ratio (OR) was 4.11 (95\% Cl: 1.79-9.44). In contrast, no difference emerged for conditions at higher risk of infection including type of job, reported direct contacts or cohabitation with persons at risk (Table 4).

Pregnancy outcome in women with and without Covid-19 diagnosis is shown in Table 5. No statistically significant differences emerged. Only one woman with Covid-19 underwent cesarean delivery because of pneumonia and severe respiratory insufficiency. After delivery, she required admission in the intensive care unit. She has now recovered. No other women needed intensive care. No maternal deaths occurred.

\section{Discussion}

Covid-19 in pregnancy was not rare in our area during the first outbreak of Sars-Cov-2. One in 11 women $(8.9 \%)$ actually entered in contact with the virus, less than estimated a priori. On the other hand, the clinical course of the disease appeared mostly unremarkable. Sixty-one percent did not report any symptom, preterm delivery because of Covid-19 maternal complications was necessary only in one case and pregnancy outcome was not markedly influenced.

Interestingly, the rate of infected women observed in our study is very similar to the prevalence observed in a concomitant survey performed in our area and focusing on blood donors. Specifically, Valenti et al. evaluated the presence of antibodies against the nucleocapsid protein and reported for April 2020 a prevalence of $7.1 \%$ (95\% Cl: $4.4-10.8 \%)$, thus in line with our findings [12]. In contrast, our reassuring clinical findings are somehow in disagreement with recent evidence from large case series of affected pregnant women. For instance, according to a recent systematic review of the literature, the rate of asymptomatic women was only $14.5 \%, 19 \%$ of affected women required delivery due to Covid- 19 related reasons, $18.5 \%$ required oxygen support and preterm birth occurred in $21.5 \%$ of cases [7]. As already pointed out in the introduction, the most plausible explanation for the discrepancy with our findings is a selection bias. The denominator is radically different. By mainly focusing on the presence of antibodies and excluding referred cases, we were able to study an unselected population. In contrast, published case series reported on women who were mainly identified because of Covid-19 related symptoms. Our data could better reflect the real impact of Covid-19 in pregnancy. Nonetheless, we cannot definitely rule out that differences in the characteristics of the population, local environmental conditions and genetic 
variants of the virus may have also impacted on clinical relevance and could play a role in explaining these inconsistencies.

Some limitations of our study should be acknowledged. Firstly, the reliability of the diagnostic tests is still a source of debate. Even if Covid-19 was investigated using three different modalities, the accuracy of all the tests used is yet uncertain. The nasopharyngeal swab could detect only ongoing infections and the sensitivity in affected cases was reported to be only $63 \%$ [13]. Preliminary evidence is comforting for the other two tests employed to detect antibodies against Sars-Cov-2 but available studies for validation are not optimal. In particular, no attempts have yet been made to investigate the accuracy of these tests for asymptomatic or poorly symptomatic cases. Inferring results obtained in patients with moderate or severe forms of the disease to the whole population is arguable. More in general, the clinical and biological significance of the different type of antibodies remain to be ascertained $[10,14,15]$. Noteworthy, in our experience, agreement between the two tests was not excellent since only 17 women were found to be positive to both tests while 7 were positive only to one of the two.

Secondly, even if women referred from other hospitals because of Covid-19 were excluded, we cannot rule out some other selection biases. On one hand, some healthy women with unremarkable history may have decided to deliver in other hospitals to avoid an Institution with Covid-19 affected cases while, on the other hand, some women with mild symptoms suggestive for the infection could have been more likely to refer to our hospital. Both biases could lead to overestimate the detected frequency of Covid-19. However, the high proportion of asymptomatic cases tends to rule out a major role of these confounders.

Finally, since Covid-19 related symptoms were retrospectively collected, one cannot exclude a recall bias. In this regard, it has however to be underlined that women were blinded to the results of the antibodies tests when interviewed and that the investigated period of time was limited to only three months. Even if episodes of mild symptoms could be overlooked, it is unlikely that more significant health troubles could be omitted.

\section{Conclusions}

Women in the second half of pregnancy do not appear to be more susceptible to Covid-19; the observed prevalence overlaps with the non-pregnant population of the same area. In addition, the study suggests that the course of the disease in the second half of pregnancy is mostly unremarkable. However, this latter observation warrants confirmation in larger studies.

\section{Abbreviations}

$\mathrm{Cl}$

Confidence Interval

CLIA

Chemiluminescence 
Covid-19

Coronavirus Infection dDisease - 19

ECLIA

Electrochemiluminescence

$\mathrm{ICH} / \mathrm{GCP}$

International Conference of Harmonization of Good Clinical Practice

$\lg G$

Immunoglobuline-type G

IRCCS

Istituto di Ricerca e Cura a Carattere Scientifico

OR

Odds Ratio

RT-PCR

multiplex Real-Time in Polymerase Chain Reaction

SARS-Cov-2

Severe Acute Respiratory Syndrome Coronavirus type-2

\section{Declarations}

Ethical approval and consent to participate: The study was approved by the local Institutional Review Board (Comitato Etico Milano Area 2, N. 356_2020). All participating women provided a written informed consent to participate.

Consent to publish: Authors, patients and local authorities do not have any impediment to allow the publication of these data.

Availability of data and material. The database can be provided on request.

Competing interests: None of the authors have any competing interest to declare.

Fundings: the study was supported by local funds of the hospital.

Authors' contributions: MR implemented the study, data collection and corrected the draft. ES designed the study and wrote the first draft, statistical analyses. BT implemented the study, supervision, corrected the draft. LLP collaborated in data collection and corrected the draft. SUR collaborated in designing the study, laboratory analyses. GB implemented the study, supervision, and corrected the draft. GL collaborated in designing the study, laboratory analyses. EF designed the study, discussed and corrected the draft.

Acknowledgments: We are in debt with Alessia Borgo and Elena Rossi and the whole team of midwives of the involved obstetrics wards for the precious collaboration in running the study. 


\section{References}

1. Grasselli G, Pesenti A, Cecconi M. Critical Care Utilization for the COVID-19 Outbreak in Lombardy, Italy: Early Experience and Forecast During an Emergency Response. JAMA. 2020;323:1545-6.

2. Gandhi RT, Lynch JB, Del Rio C. Mild or Moderate Covid-19. N Engl J Med. 2020;383:1757-66.

3. Grasselli G, Zangrillo A, Zanella A, Antonelli M, Cabrini L, Castelli A, Cereda D, Coluccello A, Foti G, Fumagalli R, lotti G, Latronico N, Lorini L, Merler S, Natalini G, Piatti A, Ranieri MV, Scandroglio AM, Storti E, Cecconi M, Pesenti A. COVID-19 Lombardy ICU Network, Baseline Characteristics and Outcomes of 1591 Patients Infected With SARS-CoV-2 Admitted to ICUs of the Lombardy Region, Italy. JAMA. 2020;323:1574-81.

4. Guan WJ, Ni ZY, Hu Y, Liang WH, Ou CQ, He JX, Liu L, Shan H, Lei CL, Hui DSC, Du B, Li LJ, Zeng G, Yuen KY, Chen RC, Tang CL, Wang T, Chen PY, Xiang J, Li SY, Wang JL, Liang ZJ, Peng YX, Wei L, Liu Y, Hu YH, Peng P, Wang JM, Liu JY, Chen Z, Li G, Zheng ZJ, Qiu SQ, Luo J, Ye CJ, Zhu SY, Zhong NS. China Medical Treatment Expert Group for Covid-19. Clinical Characteristics of Coronavirus Disease 2019 in China. N Engl J Med. 2020;382:1708-20.

5. Chen L, Li Q, Zheng D, Jiang H, Wei Y, Zou L, Feng L, Xiong G, Sun G, Wang H, Zhao Y, Qiao J. Clinical Characteristics of Pregnant Women with Covid-19 in Wuhan, China. N Engl J Med. 2020;382:e100.

6. Crovetto F, Crispi F, Llurba E, Figueras F, Gómez-Roig MD, Gratacós E. Seroprevalence and presentation of SARS-CoV-2 in pregnancy. Lancet. 2020;396:530-1.

7. Khalil A, Kalafat E, Benlioglu C, O'Brien P, Morris E, Draycott T, Thangaratinam S, Le Doare K, Heath P, Ladhani S, von Dadelszen P, Magee LA. SARS-CoV-2 infection in pregnancy: A systematic review and meta-analysis of clinical features and pregnancy outcomes. EClinicalMedicine. 2020 Aug;25:100446.

8. Figueiro-Filho EA, Yudin M, Farine D. COVID-19 during pregnancy: an overview of maternal characteristics, clinical symptoms, maternal and neonatal outcomes of 10,996 cases described in 15 countries. J Perinat Med. 2020;48:900-11.

9. Chi J, Gong W, Gao Q. Clinical characteristics and outcomes of pregnant women with COVID-19 and the risk of vertical transmission: a systematic review. Arch Gynecol Obstet. 2020 Dec 1:1-9. Epub ahead of print.

10. French MA, Moodley Y. The role of SARS-CoV-2 antibodies in COVID-19: Healing in most, harm at times. Respirology. 2020;25:680-2.

11. Tassis B, Lunghi G, Frattaruolo MP, Ruggiero M, Somigliana E, Ferrazzi E. Effectiveness of a COVID19 screening questionnaire for pregnant women at admission to an obstetric unit in Milan. Int $\mathrm{J}$ Gynaecol Obstet. 2020;150:124-6.

12. Valenti L, Bergna A, Pelusi S, Facciotti F, Lai A, Tarkowski M, Berzuini A, Caprioli F, Santoro L, Baselli G, Della Ventura C, Erba E, Bosari S, Galli M, Zehender G, Prati D. SARS-CoV-2 seroprevalence trends in healthy blood donors during the COVID-19 Milan outbreak. medRxiv [submitted].

13. Wang W, Xu Y, Gao R, Lu R, Han K, Wu G, Tan W. Detection of SARS-CoV-2 in Different Types of Clinical Specimens. JAMA. 2020;323:1843-4. 
14. Mallapaty S. Will antibody tests for the coronavirus really change everything? Nature. 2020;580:5712.

15. Sethuraman N, Jeremiah SS, Ryo A. Interpreting Diagnostic Tests for SARS-CoV-2. JAMA. 2020;323:2249-51.

\section{Tables}

Table 1. Prevalence of women who had Covid-19 in pregnancy in the studied cohort $(n=315)$ according to type of testing.

\begin{tabular}{|lccc|}
\hline Diagnostic test & N & Rate & $95 \% \mathrm{Cl}$ \\
\hline Naso-pharyngeal swab RT-PCR & 13 & $4.1 \%$ & $2.4-6.9 \%$ \\
\hline Antibodies againts CoV-2 envelope & 20 & $6.4 \%$ & $4.2-9.6 \%$ \\
\hline Antibodies againts CoV-2 nucleoprotein & 21 & $6.7 \%$ & $4.4-10.0 \%$ \\
\hline & & & \\
\hline At least one & 28 & $8.9 \%$ & $6.2-12.5 \%$ \\
\hline & & & \\
\hline
\end{tabular}

Table 2. Distribution of tests results among Covid-19 cases $(n=28)$

\begin{tabular}{|c|c|c|c|}
\hline \multicolumn{3}{|c|}{ Positive test } & \multirow[t]{2}{*}{$\mathrm{N}(\%)$} \\
\hline RNA-PCR & Anti-envelope & Anti-nucleoprotein & \\
\hline+ & - & - & $4(14 \%)$ \\
\hline- & + & - & $3(11 \%)$ \\
\hline- & - & + & $3(11 \%)$ \\
\hline+ & + & - & $0(0 \%)$ \\
\hline+ & - & + & $1(4 \%)$ \\
\hline- & + & + & $9(32 \%)$ \\
\hline+ & + & + & $8(28 \%)$ \\
\hline
\end{tabular}


Table 3. Baseline characteristics of women who did and did not test positive for Covid-19

\begin{tabular}{|llll|}
\hline Characteristics & Covid-19 & Controls & $\mathrm{p}$ \\
\cline { 2 - 4 } & $\mathrm{n}=28$ & $\mathrm{n}=287$ & \\
\hline Age (years) & & & \\
\hline BMI (Kg/m $\left.{ }^{2}\right)$ & $31.6 \pm 7.0$ & $34.2 \pm 5.4$ & 0.07 \\
\hline Gestational age (weeks) & $27.1 \pm 4.5$ & $26.4 \pm 4.2$ & 0.41 \\
\hline Ethnicity & $38.8 \pm 1.4$ & $38.8 \pm 2.0$ & 0.79 \\
\hline Caucasian & & & 0.08 \\
\hline Black / African & $20(71.4 \%)$ & $230(80.1 \%)$ & \\
\hline Asian & $2(7.2 \%)$ & $26(9.1 \%)$ & \\
\hline Hispanic & $0(0.0 \%)$ & $9(3.1 \%)$ & \\
\hline Smoking in pregnancy & $1(3.6 \%)$ & $12(4.2 \%)$ & 1.00 \\
\hline ART pregnancy & $1(3.6 \%)$ & $22(7.7 \%)$ & 0.71 \\
\hline Previous vaginal deliveries & $7(25.0 \%)$ & $89(31.0 \%)$ & 0.67 \\
\hline Previous cesarean sections & $5(17.9 \%)$ & $45(15.7 \%)$ & 0.79 \\
\hline Previous myomectomy & $0(0.0 \%)$ & $5(1.7 \%)$ & 1.00 \\
\hline Main medical complications & & & \\
\hline Thyroid disorders & $0(0.0 \%)$ & $22(7.7 \%)$ & 0.24 \\
\hline Autoimmune disorders & $0(0.0 \%)$ & $9(3.1 \%)$ & 1.00 \\
\hline Cardiovascular problems & $2(7.2 \%)$ & $6(2.1 \%)$ & 0.15 \\
\hline Thrombophylia & $0(0.0 \%)$ & $3(1.0 \%)$ & 1.00 \\
\hline Others & $2(7.2 \%)$ & $8(2.8 \%)$ & 0.22 \\
\hline Multiple pregnancy & $1(3.6 \%)$ & $10(3.5 \%)$ & 1.00 \\
\hline Flu vaccination in pregnancy & $9(32.1 \%)$ & $96(33.4 \%)$ & 1.00 \\
\hline ART: Assisted Reproductive Techniques & & \\
\hline
\end{tabular}


Table 4. Symptoms and risk factors in women who did and did not test positive for Covid-19

\begin{tabular}{|c|c|c|c|}
\hline \multirow[t]{2}{*}{ Symptoms or risk conditions } & Covid-19 & Controls & \multirow[t]{2}{*}{$\mathrm{p}$} \\
\hline & $\mathrm{n}=28$ & $\mathrm{n}=287$ & \\
\hline \multicolumn{4}{|l|}{ Symptoms suggestive for Covid-19 } \\
\hline Fever & $5(17.9 \%)$ & $13(4.5 \%)$ & 0.015 \\
\hline Cough & $7(25.0 \%)$ & $19(6.6 \%)$ & 0.004 \\
\hline Sore throat & $3(10.7 \%)$ & $17(5.9 \%)$ & 0.40 \\
\hline Rhinitis & $4(14.3 \%)$ & $19(6.6 \%)$ & 0.14 \\
\hline Headache & $0(0.0 \%)$ & $0(0.0 \%)$ & n.a. \\
\hline Diarrhea & $0(0.0 \%)$ & $2(0.7 \%)$ & 1.00 \\
\hline Vomit & $1(3.6 \%)$ & $2(0.7 \%)$ & 0.24 \\
\hline Dyspnea & $3(10.7 \%)$ & $0(0.0 \%)$ & 0.001 \\
\hline Asthenia & $0(0.0 \%)$ & $2(0.7 \%)$ & 1.00 \\
\hline Myalgias & $0(0.0 \%)$ & $2(0.7 \%)$ & 1.00 \\
\hline Anosmia & $4(14.3 \%)$ & $1(0.3 \%)$ & $<0.001$ \\
\hline Ageusia & $2(7.1)$ & $4(1.4 \%)$ & 0.09 \\
\hline Episodes of symptoms suggestive for Covid-19 & & & 0.001 \\
\hline None & $17(60.7 \%)$ & $248(86.5 \%)$ & \\
\hline 1 & $10(35.7 \%)$ & $38(13.2 \%)$ & \\
\hline 2 & $1(3.6 \%)$ & $1(0.3 \%)$ & \\
\hline \multicolumn{4}{|l|}{ Risk conditions } \\
\hline Job (health worker, lab technician) & $0(0.0 \%)$ & $3(1.0 \%)$ & 1.00 \\
\hline Direct contact with Covid-19 affected persons & $1(3.6 \%)$ & $3(1.0 \%)$ & 0.31 \\
\hline Cohabitant with persons at risk & $3(10.7 \%)$ & $31(10.8 \%)$ & 1.00 \\
\hline
\end{tabular}

Table 5. Pregnancy outcome in women who did and did not test positive for Covid-19 


\begin{tabular}{|llll|}
\hline Characteristics & Covid-19 & Controls & \multirow{2}{*}{$\mathrm{p}$} \\
\cline { 2 - 3 } & $\mathrm{n}=28$ & $\mathrm{n}=287$ & \\
\hline Gestational Diabetes Mellitus & $3(10.7 \%)$ & $22(7.7 \%)$ & 0.48 \\
\hline Hypertensive disorders & $2(7.1 \%)$ & $6(2.1 \%)$ & 0.15 \\
\hline Intrahepatic cholestasis & $1(3.6 \%)$ & $5(1.7 \%)$ & 0.43 \\
\hline Preterm delivery (<37 weeks' gestation) & $2(7.1 \%)$ & $22(7.7 \%)$ & 1.00 \\
\hline Cesarean section & $10(35.7 \%)$ & $108(37.6 \%)$ & 1.00 \\
\hline SGA newborns ${ }^{a}$ & $2(7.4 \%)$ & $30(10.8 \%)$ & 0.75 \\
\hline LGA newborns ${ }^{\text {a }}$ & $1(3.7 \%)$ & $23(8.3 \%)$ & 0.71 \\
\hline Stilbirth & $0(0.0 \%)$ & $0(0.0 \%)$ & n.a. \\
\hline Neonatal death & $0(0.0 \%)$ & $0(0.0 \%)$ & n.a. \\
\hline Maternal death & $0(0.0 \%)$ & $0(0.0 \%)$ & n.a. \\
\hline a Multiple pregnancies excluded & & & \\
\hline SGA: Small for gestational age. LGA: Large for Gestational Age. & \\
\hline
\end{tabular}

